\title{
CORRECTION
}

\section{A gene-expression score to predict obstructive CAD}

Jose D. Vargas and Joao A. C. Lima

Nat. Rev. Cardiol. 10, 243-244 (2013); published online 2 April 2013; doi:10.1038/nrcardio.2013.50

In the version of this article originally published, the sentence "The investigators also determined that the optimal number of genes to use in their GES was 19 , which had a sensitivity of $84 \% . . . "$

should have been "The investigators also determined that the optimal GES threshold was 19 , which

had a sensitivity of $84 \% . . . "$. The error has been corrected in the HTML and PDF versions of the article. 